\title{
tic\&société
}

Vol. 7, Nº 2 | 2ème semestre 2013

Mondes numériques: nouvelles perspectives de la recherche

\section{Comment articuler analyse des réseaux et des discours sur Twitter}

L'exemple du débat autour du pacte budgétaire européen

Nikos Smyrnaios and Pierre Ratinaud

\section{OpenEdition}

Journals

\section{Electronic version}

URL: http://journals.openedition.org/ticetsociete/1578

DOI: 10.4000/ticetsociete. 1578

Publisher

Association ARTIC

\section{Electronic reference}

Nikos Smyrnaios and Pierre Ratinaud, "Comment articuler analyse des réseaux et des discours sur Twitter», tic\&société [Online], Vol. 7, № 2 | 2ème semestre 2013, Online since 26 June 2014, connection on 19 April 2019. URL : http://journals.openedition.org/ticetsociete/1578; DOI : 10.4000/ ticetsociete. 1578 


\title{
Comment articuler analyse des réseaux et des discours sur Twitter: \\ L'exemple du débat autour du pacte budgétaire européen
}

\author{
Nikos SMYRNAIOS \\ Université Toulouse 3, Lerass \\ Nicolaos.smyrnaios@iut-tlse3.fr

\section{Pierre RATINAUD} \\ Université Toulouse 2, Lerass \\ Ratinaud@univ-tlse2.fr
}

Nikos SMYRNAIOS est maître de conférences en Sciences de l'information et de la communication à l'Université Toulouse 3, membre du Laboratoire d'études et de recherches appliquées en sciences sociales (LERASS) et membre associé du Groupe de recherche interdisciplinaire sur la communication, l'information et la société (GRICIS). Ses publications portent essentiellement sur les enjeux socio-économiques de l'internet. Ses principaux axes de recherches sont les stratégies des acteurs de l'information en ligne, les usages des réseaux socionumériques et particulièrement de Twitter et les pratiques journalistiques contemporaines.

Pierre RATINAUD est maître de conférences en Sciences de l'Education au département des sciences de l'éducation et de la formation de l'université de Toulouse 2 - Le Mirail et membre du Laboratoire d'études et de recherches appliquées en sciences sociales (LERASS). Ses travaux portent sur la dynamique des systèmes de représentations sociales et professionnelles, les modèles de pensée sociale et de pensée professionnelle et les analyses statistiques de données textuelles. II développe du logiciel libre de lexicométrie IRaMuTeQ (http://www.iramuteq.org). 
Comment articuler analyse des réseaux et des discours sur Twitter:

L'exemple du débat autour du pacte budgétaire européen

\title{
Comment articuler analyse des réseaux et des discours sur Twitter: \\ L'exemple du débat autour du pacte budgétaire européen
}

\begin{abstract}
Résumé : Notre article propose de repérer des communautés d'usagers au sein de Twitter et de les articuler avec les principales thématiques mobilisées dans le cadre du débat qui a eu lieu en France concernant la ratification du Traité européen sur la stabilité, la coordination et la gouvernance (TSCG) à l'automne 2012. Nos résultats montrent que les échanges mêlent des messages informatifs avec une gamme d'expressions diverses (ironie, critique, humour, indignation etc.). Le débat est structuré et ne se limite pas à une simple juxtaposition d'expressions individuelles. Les échanges sont dominés par ceux qui adoptent une position de forte opposition au Traité, couplée souvent à des discours de dénonciation; ces internautes se tournent vers les blogues et les médias militants et alternatifs dont ils font la promotion pour s'informer mais également pour tenter de changer l'agenda dominant.
\end{abstract}

Mots-clés : Twitter, discours, graphes, TSCG, débat.

Abstract: In this paper we propose a method for linking identifiable communities of users within Twitter with the main themes mobilized in the debate which took place in France during the autumn of 2012 regarding the ratification of the Treaty on Stability, Coordination and Governance in the Economic and Monetary Union (TSCG). Our results show that the debates combined informational messages with diverse expressions (e.g., irony, criticism, humor, indignation etc.) of interest, and that these exchanges were not limited to the mere juxtaposition of individual expressions. The debates were dominated by individuals who voiced strong opposition to the Treaty, and who engaged with blogs and alternative media to both inform themselves and to try to change the dominant agenda.

Keywords : Twitter, discourse, graphs, TSCG, debate. 


\section{Nikos SMYRNAIOS et Pierre RATINAUD}

Resumen : Nuestro trabajo se propone un método para identificar comunidades de usuarios dentro de Twitter y vincularlos con los temas principales movilizados en el debate que tuvo lugar en Francia en relación con la ratificación del Tratado de Estabilidad, Coordinación y Gobernanza en la Unión Económica y Monetaria (TSCG) en el otoño de 2012. Nuestros resultados muestran que los debates son una mezcla de los mensajes informativos y diversas expresiones (ironía, crítica, humor, indignación, etc), hay una forma de debate estructurado y no una simple yuxtaposición de las expresiones individuales; intercambios están dominadas por los que se inclinan contra fuerte oposición, este último están recurriendo a los blogs y medios alternativos para informarse sino también para tratar de cambiar la agenda dominante.

Palabras claves : Twitter, discurso, gráficos, TSCG, debate 
Comment articuler analyse des réseaux et des discours sur Twitter : L'exemple du débat autour du pacte budgétaire européen

La montée en puissance des réseaux socionumériques $(\mathrm{RSN})^{1}$ depuis le milieu des années 2000 en fait désormais des outils à usage massif. Des services comme Facebook et Twitter disposent de centaines de millions de membres dans le monde et leurs usages quotidiens sont infiniment complexes et variés. Pour le chercheur qui s'intéresse aux pratiques et aux représentations sociales (Moscovici, 1961), à la formation des opinions et des réseaux relationnels (Bidart, Degenne et Grossetti, 2011), ces outils proposent une foule de données. Ces dispositifs génèrent des traces numériques à chaque fois qu'un usager s'exprime, qu'il noue des relations ou qu'il échange des informations.

Ces traces existent parce que Facebook et Twitter sont avant tout des services informatiques dont le fonctionnement implique le stockage de nombreuses informations. De ce fait, ils se prêtent à des analyses quantitatives et automatisées qui portent souvent sur des grands ensembles de données (voir par exemple Cha et al., 2010). Le principal intérêt de ce type d'analyse est son caractère englobant qui permet d'avoir une vision d'ensemble de grands pans des réseaux en question. Néanmoins, du point de vue des sciences humaines et sociales, ces approches sont insuffisantes car elles ont tendance à réduire l'épaisseur du social à des mesures quantitatives relativement simplistes (Rebillard, 2011).

Le courant de recherche des Digital Methods, au sein de l'université d'Amsterdam, est une des initiatives qui tentent de remédier à cette faiblesse. Pour son initiateur Richard Rogers, les traces laissées par les internautes lors de leurs activités en ligne nous renseignent sur des pratiques sociales réelles (Rogers, 2009). Le courant des Digital Methods, à l'image de certains travaux publiés notamment dans la revue Réseaux en France, essaie ainsi de dépasser le paradigme, dominant jusqu'au début des années 2000, qui envisageait l'internet comme un espace virtuel séparé distinctement du monde social "réel ». Pour ce faire, Rogers (ibid.) affirme la nécessité de combiner des méthodes d'enquête traditionnelles en sciences sociales (entretiens, questionnaires, analyses de discours, etc.) avec des méthodes de collecte et de traitement de grands ensembles de données disponibles sur l'internet. L'une des pistes les plus fécondes dans cette approche est la mise en œuvre d'analyses de graphes afin de déceler les relations qui se développent entre usagers des réseaux socionumériques. Le rapprochement de la théorie mathématique des graphes et des sciences sociales, apparu dans les années

\footnotetext{
${ }^{1}$ Nous utilisons l'expression RSN, inspirée des travaux de Boyd et Ellison (2007) parce qu'elle met l'accent sur la nature réticulaire de ces dispositifs de communication et sur leurs caractéristiques génériques par delà leurs spécificités.
} 


\section{Nikos SMYRNAIOS et Pierre RATINAUD}

50 (Watts, 2004) et qui s'est développé principalement autour de l'analyse structurale des réseaux, prend un nouvel essor depuis que Facebook et Twitter sont devenus extrêmement populaires.

Cependant, si l'analyse de graphes nous renseigne sur la structure des relations que les internautes nouent entre eux sur les RSN, elle laisse de côté le contenu de leurs échanges. II existe bien des travaux qui se focalisent sur ce qui est dit au sein des services comme Twitter, mais même s'ils portent au départ sur de gros échantillons, ils sont souvent parcellaires sur le plan de l'analyse de discours (par exemple les vingt messages les plus retweetés sur un sujet donné, Rieder, 2012). Or, les méthodes d'aspiration des «flux » d'échanges dans les RSN permettent de capter à la fois la structure des réseaux relationnels qui s'y développent et le contenu des messages échangés à une échelle de masse. De ce fait, si l'analyse qualitative des discours tenus au sein des RSN comporte un intérêt certain pour la compréhension des faits sociaux qui s'y jouent, elle est en décalage par rapport à la très grande quantité de données disponibles.

Partant de ce constat, l'objectif de notre article est de proposer une méthodologie innovante qui vise à articuler une analyse de graphes avec un traitement lexicométrique des messages en circulation en intégrant la temporalité de leur production et de leur diffusion. Autrement dit, notre objectif est de mettre en place un protocole d'analyse permettant de repérer des communautés ${ }^{2}$ d'usagers au sein de Twitter - constituées à partir d'interactions en ligne - et, en même temps, de mettre en évidence, de manière dynamique, les principales thématiques mobilisées par chacune de ces communautés et leurs évolutions dans le temps. Pour cela, nous avons choisi d'effectuer notre analyse à partir d'un échantillon susceptible d'apporter un éclairage sur les ressorts de l'utilisation des RSN comme moyen de communication, d'information et de mobilisation dans un contexte politique. Nous nous intéressons davantage aux acteurs (présents dans l'espace public) et aux discours qui sont partie prenante de la sphère publique qu'à la sociabilité ordinaire telle qu'elle s'étudie, notamment par la sociologie des réseaux, bien que ces deux facettes soient difficilement dissociables sur les RSN (Baym et Boyd, 2012).

Dans un premier temps, nous allons effectuer une revue de la littérature relative aux travaux qui ont un point de départ similaire au nôtre, autrement dit

\footnotetext{
${ }^{2}$ La notion de "communauté " est employée ici dans le sens de la théorie des graphes. Les communautés repérées dans les graphes peuvent correspondre à différents types de collectifs humains ou à d'autres types d'agrégats en fonction de la nature des données. Pour une discussion détaillée autour du concept de communauté pour caractériser la sociabilité en ligne, voir Rieder (2010).
} 
Comment articuler analyse des réseaux et des discours sur Twitter : L'exemple du débat autour du pacte budgétaire européen

qui appréhendent le rôle des RSN dans des situations de débat médiatique et de mobilisation politique. Cet état de l'art sera particulièrement orienté vers les problèmes méthodologiques et épistémologiques que posent ces nouveaux objets d'étude. Dans un deuxième temps, nous allons restituer rapidement le contexte particulier de notre étude, à savoir le débat qui a eu lieu en France concernant la ratification du Traité européen sur la stabilité, la coordination et la gouvernance (TSCG) à l'automne 2012. Dans un troisième temps, nous allons présenter en détail la méthodologie que nous avons employée. Enfin, nous dresserons un tableau des résultats nous permettant d'attester, a posteriori, de la validité de la méthodologie proposée. Étant donné le caractère exploratoire de notre travail, les résultats présentés seront envisagés davantage comme un moyen de tester la méthodologie mise au point que pour leur signification per se.

\section{Twitter comme terrain de recherche}

La première question qui peut être posée concernant nos choix méthodologiques est celle d'avoir choisi Twitter comme terrain et objet d'étude. II existe d'autres RSN dignes d'intérêt pouvant aussi prétendre au même statut. Facebook notamment est beaucoup plus populaire auprès d'une population davantage diversifiée. Le choix de Twitter repose alors sur une double motivation. La première est d'ordre pratique. La grande majorité des messages postés sur Twitter étant publics, leur collecte et leur traitement automatique via $\mathrm{I}^{\prime} \mathrm{API}^{3}$ du service est facilitée. À l'inverse, les espaces d'échange sur Facebook sont davantage cloisonnés et l'accès conditionné par l'autorisation délivrée par tel ou tel membre à devenir son "ami ». II est donc difficile d'avoir une vision transversale des réseaux relationnels ou informationnels qui s'y forment. On entrevoit déjà les contraintes liées aux caractéristiques des différentes plateformes et leurs incidences sur les choix méthodologiques, sur lesquels nous reviendrons plus loin.

La seconde raison qui motive notre choix est corrélative à la première. Twitter étant essentiellement une plateforme de diffusion publique, les contributions de ses usagers participent à l'animation de l'espace public politique au même titre que les sites d'information ou les blogues. L'utilisation intensive de Twitter par le personnel politique, les journalistes, les blogueurs, les militants politiques et les activistes en témoigne (Lotan et al., 2011). Par conséquent, le choix de Twitter comme terrain d'étude est en accord avec notre

\footnotetext{
${ }^{3}$ Selon Wikipédia, une API (Application Programming Interface) est une interface de programmation par laquelle un logiciel offre des services à d'autres logiciels.
} 
Nikos SMYRNAIOS et Pierre RATINAUD

volonté de nous intéresser aux RSN comme composants de cet espace public particulier que constitue l'internet (Cardon, 2010).

\subsection{Infomédiation sociale et négociation collective du sens des événements}

La qualité de média public de Twitter a fait émerger ces dernières années une multitude de travaux à son propos. Un premier enseignement qu'on peut tirer de leur lecture est le fait que Twitter sert de manière systématique de moyen d'accès à l'information d'actualité. En effet, en mettant à l'œuvre des processus d'infomédiation sociale ${ }^{4}$ (Smyrnaios et Rieder, 2013), les usagers de Twitter s'en servent pour partager et recevoir des liens vers des contenus externes à la plateforme, dont une grande partie est liée à l'actualité. Comme le montrent Maireder et Ausserhofer (2013), cette diffusion/réception des nouvelles s'effectue en même temps, et dans le même flot de discussions, que tout un ensemble d'autres activités (commentaires, interpellations, débats, polémiques, satyres, questionnements etc.) portant sur des évènements rapportés ou sur d'autres qui n'ont qu'un lien lointain avec ceux qui ont déclenché les échanges initiaux. Autrement dit, " la diffusion de l'information s'accompagne d'importants efforts d'interprétation, de mise en perspective, de commentaire et de critique. II s'agit là d'une négociation, mêlant l'individuel et le collectif, du sens des évènements, de leur signification politique et du positionnement moral adéquat » (Rieder et Smyrnaios, 2012, p. 138). II s'agit de la mise en pratique d'une " herméneutique de masse " (Lovink, 2012), qui vise à attribuer du sens aux événements de manière collective et à générer des consensus sur la manière dont ils doivent être envisagés.

La négociation collective du sens des évènements d'actualité est une caractéristique reconnue par les études sur la réception des produits médiatiques au moins depuis les travaux des fonctionnalistes dans les années 40 (Esquenazi, 2003). Néanmoins, elle est marquée sur Twitter par plusieurs traits particuliers liés aux caractéristiques techniques du support et à la manière dont ses usagers se l'approprient. Premièrement, le commentaire y est concomitant à la pratique de diffusion/réception de contenus d'actualité car il se fait simultanément et souvent par les mêmes instances émettrices (les usagers). Deuxièmement, le nombre de personnes engagées dans de tels échanges peut,

\footnotetext{
${ }^{4}$ L'infomédiation sociale est une interaction triangulaire entre une production et une diffusion de contenus, des plateformes de partage et des groupes d'usagers en contexte. Ce qui sert à mettre en contact une offre hétérogène et pléthorique d'information disponible sur le web et une demande éclatée de la part des publics diversifiés n'est pas un dispositif algorithmique automatisé, comme dans le cas de Google News par exemple, mais bien l'interaction entre usagers individuels à travers des plates-formes propriétaires et structurantes.
} 
Comment articuler analyse des réseaux et des discours sur Twitter :

L'exemple du débat autour du pacte budgétaire européen

simultanément, se compter en centaines, voire en milliers. La plupart d'entre elles ne se connaissent pas personnellement en dehors de l'internet mais se rencontrent en ligne en fonction des logiques complexes qui combinent des affinités sociales et thématiques. Enfin, parmi elles, on retrouve des acteurs de l'actualité ou parfois d'évènements qui sont en train d'être commentés, qu'ils soient journalistes ou personnalités publiques (politiques, artistes, sportifs, militants, etc.). Ces derniers interagissent de manière permanente et publique entre eux mais aussi avec des usagers lambda de Twitter.

\subsection{Twitter comme support d'engagement politique}

Un deuxième enseignement que nous pouvons tirer de la littérature est que Twitter permet à des communautés militantes engagées de faire émerger leur cause dans l'espace public, en dépit parfois de l'indifférence des médias dominants. Comme le remarque Vicari (2013) à propos de l'Italie, les militants tendent à investir Twitter stratégiquement en visant à inscrire leurs revendications dans l'agenda médiatique. Ils y arrivent en relayant de manière systématique des sources d'information alternatives comme des sites d'organisations politiques, des organisations non gouvernementales (ONG), mais également des blogues et des sites d'information indépendants.

Ce type de pratique est facilité par le fait que les échanges autour d'un sujet ou d'un événement donné sur Twitter se structurent autour de mots-clés appelés hashtags. Ces mots, précédés du symbole dièse (par exemple \#TSCG pour l'acronyme du Traité sur la stabilité, la coordination et la gouvernance) permettent de rechercher et de suivre les messages qui les comportent et qui, par conséquent, se réfèrent a priori au même sujet. Selon Segerberg et Bennett (2011), en dépit de sa simplicité apparente, le hashtag permet de générer des streams, c'est-à-dire des flots de messages dont le fonctionnement est complexe. Ils peuvent servir à rallier une communauté, à créer un champ de débats pour des points de vue contradictoires, à attirer l'attention du public de manière ponctuelle ou à effectuer de la veille autour d'un thème et ceci dans la durée.

\subsection{La question de la constitution d'échantillons}

Plusieurs méthodes de constitution d'échantillons, recensées notamment par Rieder (2012), s'offrent au chercheur qui s'intéresse à Twitter. Elles se fondent sur trois types génériques de collecte de données : la collecte exhaustive de toute la production de messages diffusés sur Twitter ou d'un échantillon aléatoire statistiquement correct (via la Streaming $A P I$ ); la collecte de la 


\section{Nikos SMYRNAIOS et Pierre RATINAUD}

production de messages d'un ensemble d'usagers sélectionnés en fonction de critères prédéfinis (langue, géolocalisation, graphe); enfin, la collecte de messages comportant un mot-clé spécifique. La logique et les difficultés de chaque type de collecte sont différentes.

La collecte de la totalité de messages diffusés sur Twitter est quasiment impossible aujourd'hui étant donné les contraintes qu'elle implique (autorisation, infrastructure technique nécessitant plusieurs dizaines de serveurs, voir (Cha et al., 2010). L'exploitation d'un échantillon représentatif de $1 \%$ de tous les messages diffusés sur Twitter, accessibles via la Streaming API, permet d'avoir une vision d'ensemble et statistiquement correcte du flot d'informations qui transite par le service et qui atteint aujourd'hui les 450 millions de messages par jour. Néanmoins, étant par nature disparate, mêlant différentes langues, populations et sujets de discussion, l'échantillon collecté ainsi ne peut nous renseigner sur des questions de recherche spécifiques, mais seulement dépeindre un canevas global sur lequel se déploie l'activité de ses usagers (Gerlitz et Rieder, 2013).

La collecte de la production de messages d'un ensemble d'usagers présélectionnés s'avère très utile pour étudier les pratiques et les discours d'une population spécifique. Par exemple, Rieder et Smyrnaios (2012) ont travaillé sur un échantillon de 24351 comptes d'usagers essentiellement francophones, ayant un intérêt prononcé pour l'actualité et la politique. En aspirant tous les messages produits par ces comptes pendant deux mois, ils ont pu en extraire les principaux sujets de discussion et mesurer leur importance. Le principal désavantage de cette approche est le fait que tous les messages portant sur les sujets en question mais produits par des usagers extérieurs à l'échantillon présélectionné échappent à l'analyse.

Par conséquent, si l'on veut s'intéresser à un sujet particulier, la méthode la plus adaptée est la collecte des messages comportant un ou plusieurs motsclés ou hashtags qui renvoient au sujet en question (Burgess et Bruns, 2012). $\mathrm{Ce}$ type d'analyse permet de rendre compte d'une discussion publique médiatisée sans connaître a priori les personnes qui y participent. Autrement dit, l'échantillon de comptes analysés est constitué de manière ad hoc pour chaque sujet étudié à travers une requête par mot-clé. Si cette méthode présente des avantages certains, elle pose néanmoins des problèmes méthodologiques importants. Le premier problème est celui de la représentativité. C'est une évidence d'affirmer que les usagers de Twitter ne constituent pas un groupe représentatif de la population française dans son ensemble. En effet, selon les études disponibles, le pourcentage de Français qui disposent d'un compte Twitter se situe entre $10 \%$ et $15 \%$ (Semiocast, 2012 ; Vedel, 2012). Au sein de 
Comment articuler analyse des réseaux et des discours sur Twitter : L'exemple du débat autour du pacte budgétaire européen

cette frange, il y a une surreprésentation des jeunes, des personnes diplômées et politiquement actives. II n'est donc pas possible de généraliser les observations effectuées sur Twitter à l'ensemble de la société. Étant donné les caractéristiques sociodémographiques particulières de ses usagers, il est tout à fait possible qu'une opinion ou un point de vue dominant au sein de la twittosphère francophone soit très minoritaire dans l'ensemble de la population française.

Par ailleurs, il n'est pas possible d'affirmer de manière absolue que les messages collectés à partir de mots-clés ou de hashtags sont représentatifs de l'ensemble des messages qui se réfèrent à un sujet ou un évènement particulier. II est techniquement impossible de vérifier que Twitter renvoie des résultats exhaustifs en réponse aux requêtes effectuées via son API. Mais aussi parce qu'il est facile de parler d'un même sujet avec des mots différents ou dans le cadre d'une discussion portant sur une autre thématique. De même, il faut considérer le problème de la polysémie de certains mots. Ce sont là des problèmes classiques des études lexicométriques qui persistent au niveau de l'analyse sur des corpus extraits de Twitter mais qui s'y doublent d'un autre problème: dans le cadre des études fondées sur cette méthode, les mots servent aussi à délimiter le corpus.

Une solution qui permet d'améliorer la pertinence de l'échantillon par rapport au problème de la polysémie est de choisir un terme avec un sens univoque, comme le terme TSCG sur lequel nous avons travaillé. Ce choix en revanche ne résout pas le problème de messages qui se réfèrent au sujet sans utiliser ce terme. Ceci d'autant plus que les hashtags eux-mêmes sont produits par des communautés d'usagers souvent dans un but stratégique. En l'occurrence, \#TSCG est un hashtag promu par les opposants au Traité. Par conséquent, il y a un risque de surreprésentation de ces derniers dans l'échantillon car, comme pour toute situation de controverse, l'attention est focalisée sur ceux qui y participent. L'analyse que nous présenterons par la suite est donc sujette à précautions.

\section{Le contexte politique}

Le Traité sur la stabilité, la coordination et la gouvernance au sein de l'Union économique et monétaire vise à renforcer la discipline budgétaire des États de la zone euro. II comprend un mécanisme de correction automatique en cas d'écart par rapport aux objectifs fixés. Signé le 2 mars 2012 par 25 États membres de l'Union européenne (UE) - tous sauf le Royaume-Uni et la République Tchèque -, ce nouveau traité est entré en vigueur le 1er janvier 


\section{Nikos SMYRNAIOS et Pierre RATINAUD}

2013. Pour ses défenseurs, il constitue un pas dans la direction d'une union politique renforcée au sein de l'UE. Pour ses détracteurs, il s'agit d'un texte qui institutionnalise l'austérité et impose le primat de l'Allemagne en Europe.

Signé initialement par Nicolas Sarkozy, le Traité a constitué un enjeu politique pour les élections de 2012 en France. En effet, lors de la campagne présidentielle, le candidat François Hollande avait promis qu'en cas de victoire, il ne ratifierait pas le TSCG sans renégociation en faveur de la croissance et de l'emploi. En lieu et place d'une renégociation du traité, refusée par l'Allemagne, un pacte pour la croissance et l'emploi a été adopté lors du conseil européen du 29 juin 2012 qui comprenait notamment des «mesures de croissance à effet rapide ", pour un montant de 120 milliards d'euros. La signature de ce pacte a déclenché la décision du gouvernement français de ratifier le Traité. L'Assemblée Nationale a voté la loi de ratification le 9 octobre 2012 et le Sénat le 11 octobre avec les suffrages du parti socialiste (PS), de l'union pour un mouvement populaire (UMP) et du centre. La loi a été promulguée le 22 octobre 2012.

Le Traité sur la stabilité, la coordination et la gouvernance, dont l'acronyme TSCG est employé par la presse dès la fin 2011, fera l'objet de critiques en provenance de la gauche hors PS (EELV, Parti de Gauche, PCF, NPA, LO) ${ }^{5}$ mais aussi de la part de la droite souverainiste et de l'extrême droite. Le Front de Gauche organisera même une manifestation contre la ratification du Traité le 30 septembre 2012 à Paris où se sont ressemblées plusieurs dizaines de milliers de personnes. Le débat sur Twitter portant sur cette question a été très vif dès l'été 2012 et jusqu'à la promulgation de la loi. De manière générale, il suivait le clivage politique précédemment mentionné entre d'un côté les opposants (à gauche du PS et à droite de l'UMP) et, de l'autre, les partisans (PS, UMP et centre) ; une dichotomie repérée notamment par Fouetillou (2008) au sujet du referendum sur le Traité constitutionnel européen de 2005.

\section{La méthode de collecte}

La première étape consiste à choisir l'outil permettant d'archiver les tweets contenant le terme TSCG, dans sa forme de hashtag ou pas. Or, il existe aujourd'hui un très grand nombre de solutions de mesure et d'analyse concernant Twitter, dont la majorité est constituée de logiciels propriétaires et commerciaux. Les deux méthodes généralement employées sont d'une part la

\footnotetext{
${ }^{5}$ Les acronymes correspondent respectivement aux partis politiques suivants : PS (Parti socialiste), EELV (Europe Écologie Les Verts), PCF (Parti Communiste Français), NPA (Nouveau Parti Anticapitaliste) et LO (Lutte Ouvrière).
} 
Comment articuler analyse des réseaux et des discours sur Twitter : L'exemple du débat autour du pacte budgétaire européen

recherche a posteriori dans la base de données de Twitter de tous les messages comportant un mot-clé spécifique (méthode asynchrone) et, d'autre part l'enregistrement au fur et à mesure des tweets au moment de leur production via l'une des API de Twitter (méthode synchrone).

La méthode asynchrone n'est disponible que par le biais des sociétés qui acquièrent les archives de Twitter auprès de ses deux vendeurs officiels que sont Datasift et Gnip ${ }^{6}$. Ces deux sociétés achètent ces archives et les revendent à des prestataires comme Topsy ${ }^{7}$ qui les commercialisent. La méthode asynchrone a l'avantage de permettre à l'observateur de retrouver les tweets qui comportent un hashtag longtemps après que celui-ci ait émergé. L'inconvénient est que la fiabilité de la recherche semble comparativement faible, car le risque de perte de messages est élevé ${ }^{8}$.

La méthode synchrone semble quant à elle plus fiable au niveau de la collecte mais comporte un inconvénient majeur: l'enregistrement des tweets doit démarrer relativement vite après qu'un hashtag ait émergé, sinon une partie importante du débat risque de ne pas être enregistrée. Enfin, dans les deux cas - méthode synchrone et asynchrone - l'opacité des logiciels propriétaires, dont le code source n'est pas disponible, ne permet pas de comprendre comment la collecte et l'analyse s'effectuent. Pour notre part, nous privilégions la méthode synchrone, pour des raisons de précision, et les logiciels libres pour des raisons de transparence technique ${ }^{9}$. Cependant, au moment de cette recherche, nous n'avions pas connaissance qu'une solution libre de collecte synchrone existait, nous avons donc utilisé Tweet Archivist ${ }^{10}$ pour réaliser cette étude.

De manière générale, la principale difficulté dans ce type d'exercice est la dépendance du chercheur, pour la constitution de son corpus, à des technologies complexes et opaques non seulement au niveau de la capture et éventuellement du traitement, mais aussi de la source même qui n'est autre qu'une plateforme privée soumise au bon vouloir de ses propriétaires (Smyrnaios, 2013) et sujette aux dysfonctionnements inhérents aux technologies complexes.

\footnotetext{
${ }^{6}$ En réalité la recherche de tweets passés est possible gratuitement via l'API de Twitter jusqu'à un seuil dans le temps. Au delà, l'accès aux archives est payant.

${ }^{7}$ Topsy est un service de recherche de tweets (http://topsy.com)

${ }^{8}$ Un test effectué par nos soins avec la version commercialisée de Topsy a montré que le nombre de tweets retrouvé est très inferieur à celui d'un enregistrement synchrone sur la même période et pour le même hashtag.

${ }^{9}$ Deux solutions satisfaisantes qui remplissent ces critères sont le logiciel libre TwapperKeeper et l'outil Twitter Analytics de la Digital Methods Initiative.

${ }^{10} \mathrm{http}: / / \mathrm{www}$.tweetarchivist.com/archivist-desktop.
} 


\section{Nikos SMYRNAIOS et Pierre RATINAUD}

Tweet Archivist fournit à l'utilisateur du logiciel un fichier disponible dans différents formats contenant les tweets et quelques métadonnées, dont le nom d'usager et la date du tweet. Entre le 26 septembre et le 19 octobre 2012, nous avons collecté 33276 messages comportant le terme TSCG, avec ou sans dièse ${ }^{11}$. Bien que la polémique autour de l'intention de la majorité socialiste d'adopter le Traité ait commencé durant l'été 2012, notre collecte n'a pu commencer que le 26 septembre, conditionnée par la mise en place du dispositif. Nous avons arrêté la collecte le 20 octobre, après avoir constaté une diminution drastique du nombre de tweets à ce sujet. À partir du texte des tweets, nous avons extrait plusieurs informations :

- - le fait qu'il s'agisse d'un retweet ou pas : les retweets commencent par la mention RT ;

- - le nom de l'usager retweeté s'il s'agit d'un retweet;

- - les noms des usagers éventuellement mentionnés dans le tweet ;

- - les urls (Uniform Resource Locator, c.-à-d. adresses web) qui apparaissent dans le tweet: dans Twitter, toutes les urls sont raccourcies par l'intermédiaire du service de raccourcissement propriétaire de Twitter, http://t.co. Transformer ces urls courtes dans leur forme originale représente une étape supplémentaire qui ne sera pas abordée ici ;

- - à ce stade, nous avons procédé également à un nettoyage du message original duquel nous avons retiré les noms des usagers retweetés et mentionnés, ainsi que les urls et le hashtags qui a servi à l'indexation (ce dernier est présent dans tous les tweets analysés).

Nous avons obtenu un nouveau fichier qui contient le message original, le nom de l'usager émetteur, la date, le fait qu'il s'agisse d'un retweet ou pas, le nom de l'usager retweeté, le ou les noms des usagers mentionnés, les urls contenues dans le tweet ainsi que le message sous forme nettoyée qui est utilisé dans les analyses textuelles.

\section{Temporalité de la production et usagers actifs}

Nous observons sur la figure 1 que les deux pics du nombre de tweets comportant le terme TSCG correspondent aux deux évènements les plus importants de cette période liés au sujet du Traité, à savoir la manifestation du

\footnotetext{
${ }^{11}$ A titre de comparaison, une recherche asynchrone avec la version payante de Topsy a retrouvé 24109 tweets contenant la chaîne de caractères TSCG dans la même période.
} 
Comment articuler analyse des réseaux et des discours sur Twitter :

L'exemple du débat autour du pacte budgétaire européen

30 septembre organisée par le Front de Gauche et le vote de l'Assemblée le 9 octobre. Le troisième pic le plus important est observé le 2 octobre, date du début de la discussion à l'Assemblée. Cette montée en puissance de l'intérêt du public pour le Traité est également visible dans le volume de requêtes effectuées sur Google avec le terme TSCG qui culmine également pendant la même période (figure 2).

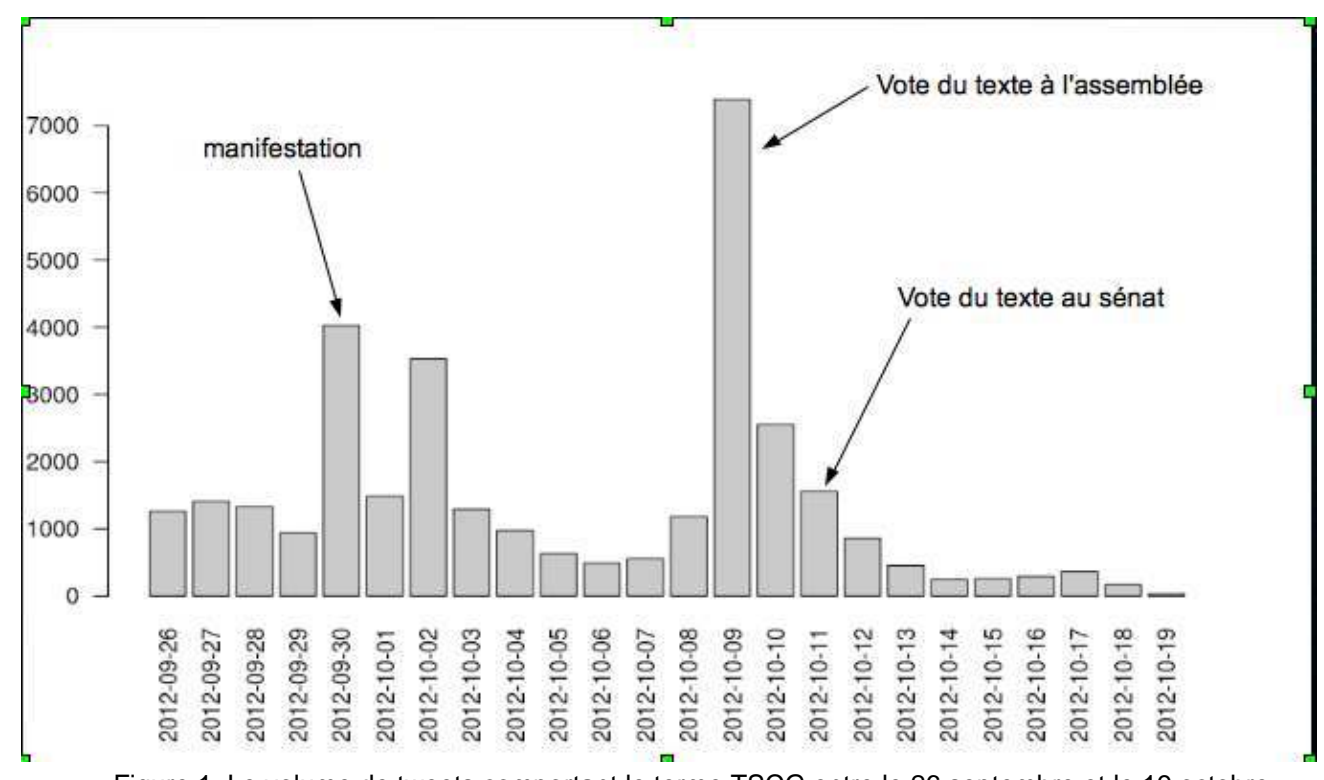

Figure 1. Le volume de tweets comportant le terme TSCG entre le 26 septembre et le 19 octobre 2012. 


\title{
Nikos SMYRNAIOS et Pierre RATINAUD
}

\author{
sept. 2012 oct. 2012 \\ Figure 2. Le volume de recherche comportant le terme TSCG entre août et novembre 2012 \\ (source : Google).
}

Une première lecture de messages de ces deux journées permet de constater que la surproduction de tweets par rapport au reste de la période est due à des messages «militants " mais de type différent : le 30 septembre, la majeure partie des messages est constituée d'appels à la mobilisation, puis de "reportages » depuis les lieux (photographies, commentaires, etc.) et enfin d'une discussion sur le succès de la manifestation et sur ses retombées politiques ; le 9 octobre en revanche, ce sont les expressions d'indignation qui prévalent, ce particulièrement envers les députés PS qui ont approuvé le texte.

En revanche, entre le 2 et le 8 octobre, pendant le débat parlementaire, outre le déploiement d'arguments essentiellement contre le Traité ainsi que de messages humoristiques et ironiques, nous trouvons aussi des tweets portant sur le déroulement des discussions à l'Assemblée, diffusées en direct par la chaîne parlementaire. II s'agit là d'un usage éminemment citoyen de Twitter puisque celui-ci est utilisé pour retransmettre et commenter en direct (livetweeting) les différentes prises de parole dans l'enceinte de la représentation nationale.

Parmi les 33276 messages recensés, 18040 (54,2\%) sont des retweets, c'est-à-dire des citations intégrales ou partielles d'autres messages. II s'agit d'un pourcentage exceptionnellement élevé par rapport à des situations comparables où la part des retweets se situe autour du tiers de l'ensemble (voir par exemple Bruns et Burgess, 2011). Ce résultat montre que la structure du débat autour du TSCG est relativement centralisée autour d'un petit nombre d'intervenants qui attire un grand nombre de citations. L'inégale répartition de l'expression publique sur Twitter est confirmée par notre échantillon qui présente une structure centralisée : sur les 7156 usagers, 3818 ont produit un seul tweet 
Comment articuler analyse des réseaux et des discours sur Twitter :

L'exemple du débat autour du pacte budgétaire européen

$(53,35 \%$ de l'ensemble) et $23 \%$ d'entre eux représentent $76,6 \%$ des messages.

Cette structure particulièrement centralisée peut être aussi expliquée par l'investissement dans le hashtag \#TSCG des opposants au traité, notamment des militants ou sympathisants du Front de Gauche (figure 3). En effet, parmi les trente usagers les plus actifs, c'est-à-dire ayant produit le plus grand nombre de tweets ou de retweets comprenant le terme en question, dix-huit déclarent une filiation à l'une des composantes du Front de Gauche dans leurs autodescriptions, six montrent une sensibilité de gauche sans déclarer une identité partisane, quatre sont proches du mouvement souverainiste «Debout la République " de Nicolas Dupont-Aignan. Euractiv (www.euractiv.fr) est, quant à lui, un média d'information européenne qui a investi aussi le hashtag \#TSCG (TSCG_est un compte de spam).
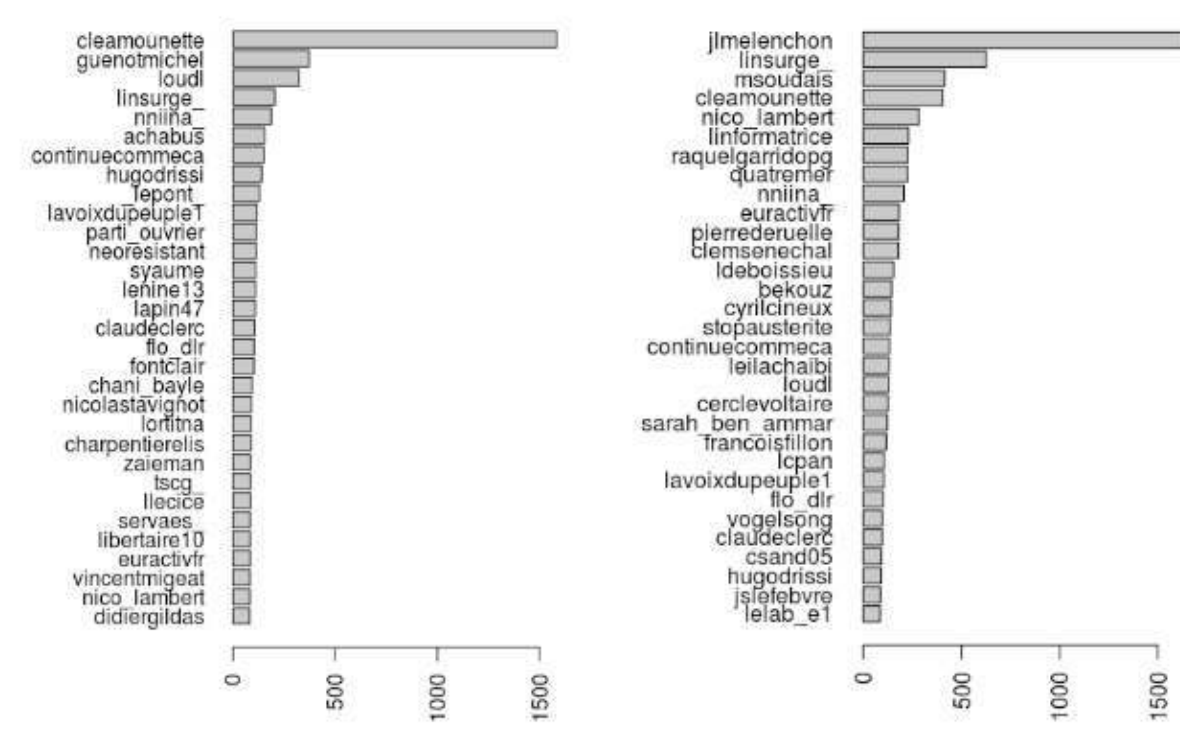

Figure 3. Les 30 usagers ayant produit le plus de tweets (à gauche) et ayant reçu le plus de retweets (à droite) comportant le terme TSCG entre le 26 septembre et le 19 octobre 2012.

À partir de ces données, il est clair que les usagers qui ont investi massivement le débat autour du Traité sur Twitter sont essentiellement des 


\section{Nikos SMYRNAIOS et Pierre RATINAUD}

opposants à celui-ci, venant en majorité de "la gauche de la gauche ", et en minorité de la droite souverainiste et de l'extrême droite. Néanmoins, la liste des trente usagers ayant reçu le plus de retweets montre une image plus nuancée. Le compte officiel de Jean-Luc Mélenchon est de loin le plus retweeté, renforçant le constat précédant, suivi d'autres cadres du Front de Gauche (PCF et Parti de Gauche) comme Raquel Garrido, Cyril Cineux ou Leila Chaïbi. Dans le même temps, nous retrouvons, bien placés dans la liste des usagers ayant une position neutre ou favorable au traité, les journalistes tels que Laurent de Boissieu (La Croix) et Jean Quatremer (Libération). Néanmoins, ce dernier journaliste, correspondant à Bruxelles bien connu dans la twittosphère française, est souvent cité pour être critiqué, parfois avec humour, par les opposants au Traité.

\section{La mise en avant d'une information alternative}

Les noms de domaine les plus mentionnés dans le flot de messages comportant le terme TSCG sont également assez révélateurs de la nature du débat. En dehors du blogue Zebuzzeon - tweeté systématiquement par l'utilisatrice Clea Mounette d'une manière qui s'apparente à une pratique de spam - on y trouve essentiellement des plateformes de partage de contenu comme Dailymotion et YouTube, ainsi que des version en ligne des médias traditionnels comme Le Monde, Le Nouvel observateur, L'Humanité et Europe 1, ce qui est assez classique sur Twitter (Rieder et Smyrnaios, 2012). Cependant, l'originalité de notre échantillon se situe au niveau de deux autres caractéristiques particulières: d'une part la recherche d'une information citoyenne «brute", incarnée notamment par la popularité du site de l'Assemblée Nationale et dans une moindre mesure celui du Sénat, et, d'autre part, l'importance des sources d'information «alternatives ", c'est-à-dire ne faisant pas partie des médias traditionnels (figure 4). Ainsi, parmi les sources les plus citées, nous retrouvons deux plateformes d'hébergement de blogues (Overblog et Wordpress) ${ }^{12}$, trois sites affiliés au Front de Gauche (Jean-lucmelenchon.fr, Lepartidegauche.fr et Placeaupeuple2012.fr), plusieurs sites web d'information natifs du web (Mediapart, Slate) et/ou participatifs (Agoravox, Bastamag) et des blogues militants (SuperNo, Stopausterité).

\footnotetext{
${ }^{12}$ Nous faisons ici référence aux noms de domaine extraits à partir des urls. II est possible d'envisager une étude plus précise des blogues mobilisés mais c'est une étape que nous n'avons pas réalisée dans le cadre de ce travail.
} 
Comment articuler analyse des réseaux et des discours sur Twitter:

L'exemple du débat autour du pacte budgétaire européen

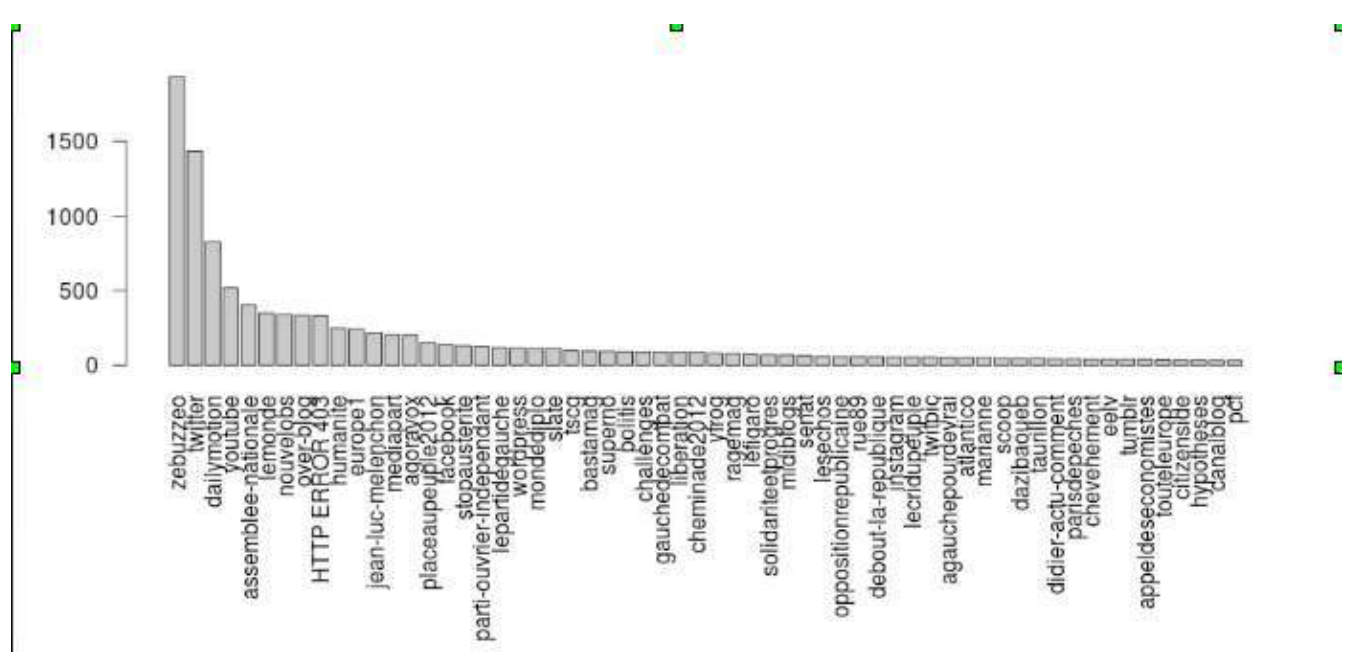

Figure 4. Les 60 sites web les plus cités dans l'échantillon.

Ces caractéristiques sont confirmées par une analyse plus fine au niveau des pages de destination permise par Topsy. II y apparaît que les cinq contenus les plus partagés dans l'échantillon des messages comportant le terme TSCG entre le 26 septembre et le 19 octobre 2012 sont dans l'ordre : la synthèse du vote publiée par le site de l'Assemblée nationale (244 tweets et retweets dans la

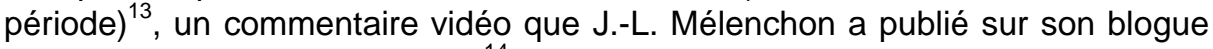
après l'adoption du Traité $(100)^{14}$, un appel à manifester le 30 septembre 2012 sur le site Place au Peuple $(87)^{15}$, une critique du Traité par l'économiste Fréderic Lordon publiée sur le blogue du Monde diplomatique $(63)^{16}$ et un article critique de l'économiste Cédric Durand publié par le pure player de gauche Bastamag $(61)^{17}$.

II apparaît donc clairement que les usagers de Twitter participent au débat non seulement via les commentaires qu'ils produisent et les opinions qu'ils

\footnotetext{
${ }^{13} \mathrm{http}: / /$ www.assemblee-nationale.fr/14/scrutins/jo0030.asp.

14 http://www.jean-luc-melenchon.fr/2012/10/09/commentaires-de-j-I-melenchon-apres-ladoption-duTSCG/.

${ }_{15}^{15} \mathrm{http}: / /$ www.placeaupeuple.fr/manifestation-du-30-septembre/.

${ }^{16} \mathrm{http}: / /$ blog.mondediplo.net/2012-09-26-En-sortir.

${ }^{17} \mathrm{http}: / /$ www.bastamag.net/TSCG-vrais-mensonges-fausses.
} 


\section{Nikos SMYRNAIOS et Pierre RATINAUD}

émettent mais aussi à travers les liens qu'ils partagent. Les opposants au Traité, ne trouvant apparemment pas satisfaction auprès des médias traditionnels, considérés comme trop proches de la ligne officielle, se tournent vers les blogues et les médias militants et alternatifs. L'objectif est bien sûr de s'informer mais également peut-être de tenter de changer l'agenda des médias en mettant en avant un traitement différent de cette actualité.

\section{Articulant communautés et classes de discours}

Afin de mieux comprendre la structure du débat autour du Traité, nous avons appliqué à l'échantillon collecté une méthode d'analyse qui repose dans un premier temps sur l'étude de graphes de relations entre usagers. Nous avons construit un graphe des interactions entre usagers à partir de la pratique du retweet de la manière suivante: pour chaque retweet de l'échantillon, nous traitons l'usager émetteur et l'usager retweeté comme les sommets d'un graphe. Le nombre de fois où le couple émetteur/retweeté apparaît (dans cet ordre) donnera l'ordre de grandeur des arêtes qui relient les sommets. Le graphe ainsi obtenu est donc un graphe orienté par une forme de relations entre usagers de Twitter qui ont inclus dans leurs messages le terme TSCG pendant la période étudiée.

Ce choix a été fondé sur des travaux (Cha et al., 2010, Rieder et Smyrnaios, 2012) qui ont montré que les retweets et les mentions étaient les meilleurs indicateurs du poids d'une relation entre usagers dans le cadre d'un sujet de débat spécifique. Le fait de se suivre (follower et friend) obéit à des logiques différentes. De cette manière, nous avons pu constituer le graphe reliant les usagers de notre échantillon. L'étape suivante a consisté à déterminer des communautés à l'intérieur de ce graphe, c'est-à-dire des ensembles de sommets fortement reliés entre eux, ce qui revient dans notre exemple à déterminer des groupes d'individus qui ont tendance à se retweeter. Nous avons utilisé ici l'algorithme proposé par Blondel et al. (2008) et qui est disponible dans le logiciel $\mathrm{Gephi}^{18}$. Sur les quinze communautés détectées par l'algorithme, nous n'avons retenu que les six plus importantes (en couleurs différentes sur la figure 5).

\footnotetext{
${ }^{18}$ Gephi est un logiciel libre disponible à l'adresse http://gephi.org/.
} 
Comment articuler analyse des réseaux et des discours sur Twitter :

L'exemple du débat autour du pacte budgétaire européen

Figure 5. Le graphe global du terme TSCG basé sur les retweets.

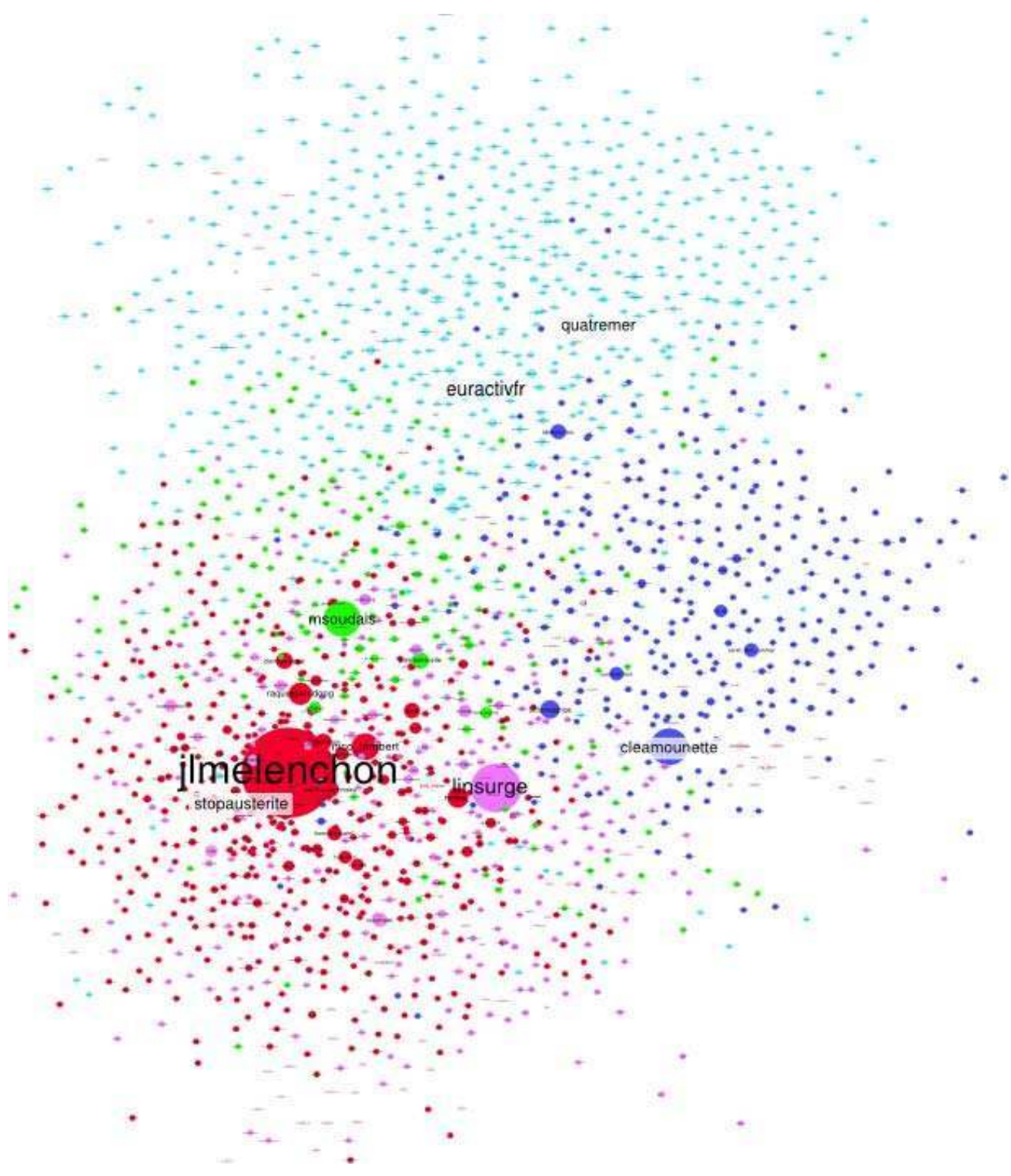




\section{Nikos SMYRNAIOS et Pierre RATINAUD}

La taille des points et des labels est proportionnelle au nombre de fois où l'usager a été retweeté. Les labels Stopaustérité, Euractiv et Quatremer ont été grossis manuellement pour la lisibilité ${ }^{19}$.

Après avoir examiné manuellement la composition de ce graphe et la répartition des nœuds et des liens, nous avons constaté que l'algorithme de détection de communautés a été un moyen très efficace pour repérer les groupes d'usagers ayant les mêmes affinités politiques. Nous avons nommé ces communautés par le pseudonyme d'un des usagers les plus fréquemment retweetés. Le tableau 1 présente les tailles respectives de chacune des communautés.

\begin{tabular}{|l|l|}
\hline Nom de la communauté & Taille (en pourcentage des usagers) \\
\hline Quatremer & $31,7 \%$ \\
\hline Melenchon & $23,74 \%$ \\
\hline $\begin{array}{l}\text { Cleamounette } \\
\text { Msoudais }\end{array}$ & $16,55 \%$ \\
\hline Linsurgé & $8,57 \%$ \\
\hline Stopaustérité & $8,42 \%$ \\
\hline \multicolumn{1}{|c|}{ Tableau 1. Les communautés de \#TSCG. } & $5,62 \%$ \\
\hline
\end{tabular}

Ainsi, le graphe est dominé par quatre groupes d'usagers qui s'agrègent respectivement autour du compte officiel de Jean-Luc Mélenchon, deux comptes des militants du Front de gauche, L'insurgé et Stop Austérité, et celui de Michel Soudais, journaliste à Politis. Ces quatre groupes sont constitués par des opposants au Traité, sympathisants de gauche (sous-graphes à gauche et vers le bas de la carte de couleur rouge, rose et vert). Cet ensemble représente $46,35 \%$ des usagers de l'échantillon.

Nous trouvons ensuite un groupe beaucoup plus petit $(16,55 \%$ des usagers), constitué des sympathisants de droite et d'extrême droite opposants au Traité et agrégés autour du compte de Clea Mounette (à droite et vers le bas de la carte, de couleur bleu foncé). Enfin, un groupe assez hétérogène et relativement dispersé s'organise autour de Euractiv et de Jean Quatremer (au

\footnotetext{
${ }^{19}$ Une version interactive de ce graphe est disponible à l'adresse : http://www.iramuteq.org/xyz/tscg.
} 
Comment articuler analyse des réseaux et des discours sur Twitter : L'exemple du débat autour du pacte budgétaire européen

centre et vers le haut de la carte, de couleur bleu clair). Ces usagers représentent $31,7 \%$ de l'échantillon. Ils sont plutôt favorables au Traité, ou en tout cas sceptiques par rapport à la farouche opposition de deux autres groupes. Nous pouvons vérifier à travers cette analyse que le critère choisi pour constituer ce graphe (les retweets) est pertinent pour dessiner des communautés d'usagers qui représentent des groupes ayant un certain type d'affinités (en l'occurrence politiques).

Afin de lier ces groupes aux discours qu'ils produisent dans Twitter, nous avons extrait, pour chaque usager, la communauté à laquelle il appartient. Nous avons utilisé cette information comme variable dans une analyse de type ALCESTE $^{20}$ (Ratinaud et Marchand, 2012) réalisée avec le logiciel Iramuteq ${ }^{21}$ (Ratinaud, 2009). Dans cette analyse, nous n'avons utilisé que les tweets originaux (ce qui revient à éliminer les retweets) ${ }^{22}$. Ainsi, la communauté d'appartenance des usagers est déterminée à partir des citations (retweets) alors que l'analyse textuelle porte sur l'information générée (tweets originaux). L'objectif est dans un premier temps de mettre en évidence les différentes thématiques abordées autour du TSCG plus que le poids qu'elles représentent dans la twittosphère. La détermination du "poids réel » de chacune d'entre elles en termes de messages produits et reproduits peut éventuellement intervenir a posteriori, en réinjectant les retweets dans l'analyse.

Concrètement, nous avons construit un corpus de textes à partir des tweets originaux des usagers appartenant à l'une des six communautés conservées. Chaque tweet est marqué par sa date et par la communauté de l'usager émetteur. Dans l'analyse ALCESTE à laquelle nous avons procédé, le vocabulaire contenu dans les tweets est lemmatisé (les verbes sont ramenés à l'infinitif, les noms au singulier et les adjectifs au masculin singulier). Seules les formes pleines (les noms, les adjectifs, les verbes et les adverbes) participent à l'analyse, les mots outils sont conservés et replacés a posteriori dans les résultats. L'objectif est de proposer une classification des tweets en fonction de la proximité du lexique qui les compose. Les classes terminales obtenues représentent donc des ensembles de tweets qui ont tendance à contenir les mêmes mots. Le corpus ainsi extrait contenait 9312 tweets. L'analyse finale propose sept classes qui regroupent $92 \%$ de ces tweets. La figure 6 résume les

\footnotetext{
${ }^{20}$ La méthode ALCESTE repose sur une classification hiérarchique descendante menée sur des segments de texte (ici les tweets). Les segments sont regroupés en fonction de leur proximité lexicale.

${ }_{21}^{21}$ IRaMuTeQ est un logiciel libre de lexicométrie (http://www.iramuteq.org).

22 Le fait de garder les retweets dans l'analyse à laquelle nous procédons aurait trop fortement orienté les résultats du point de vue de l'analyse lexicométrique.
} 


\section{Nikos SMYRNAIOS et Pierre RATINAUD}

résultats obtenus et les croise avec la temporalité d'apparition de chacune des classes.
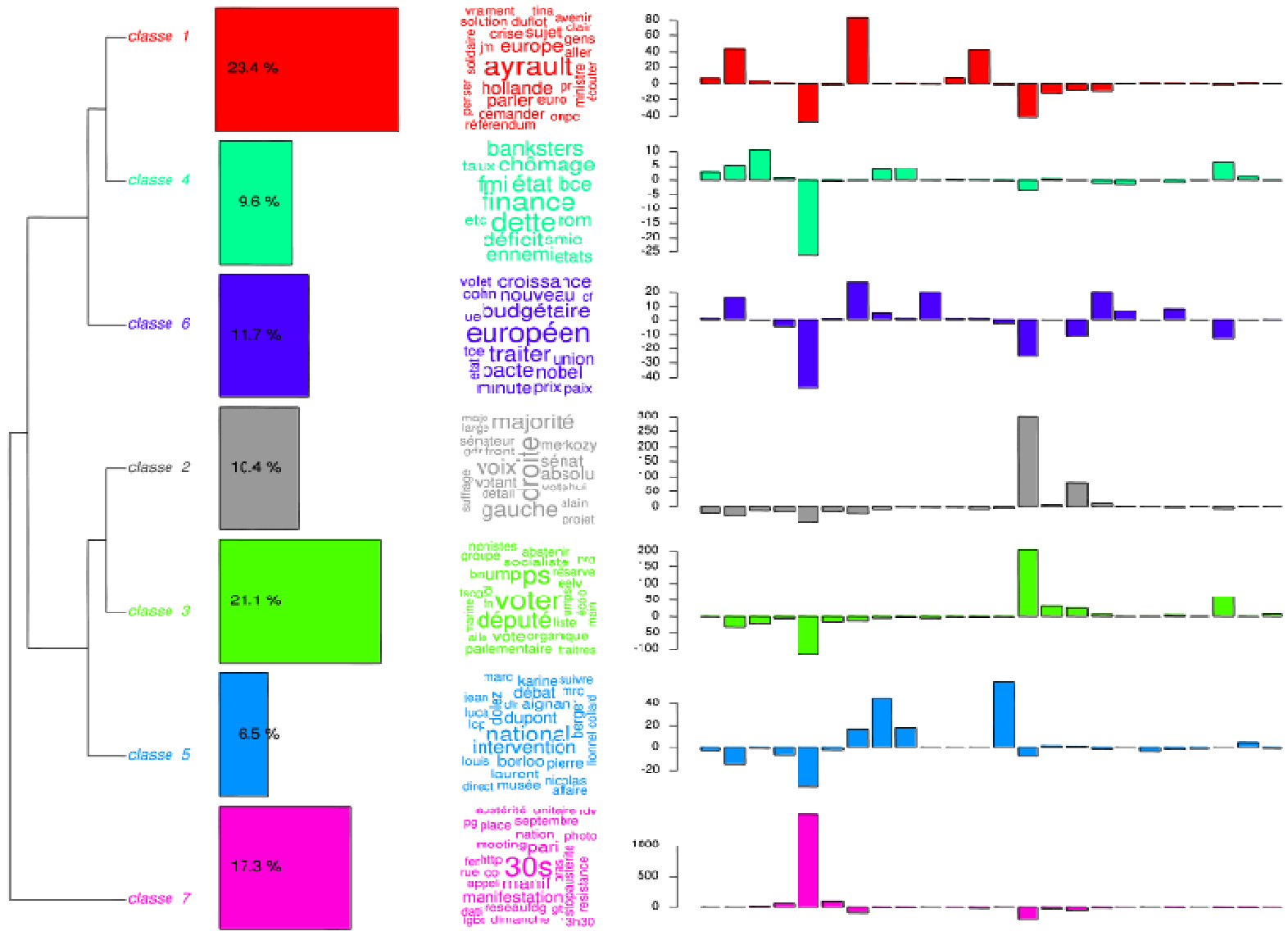

Figure 6. De gauche à droite : dendrogramme de la classification avec la taille relative de chacune des classes ; nuage des formes pleines caractéristiques de chacune des classes $^{23}$;

${ }^{23}$ Dans ces nuages de mots, la taille des mots est proportionnelle à leur chi2 d'association à la classe. 
Comment articuler analyse des réseaux et des discours sur Twitter :

L'exemple du débat autour du pacte budgétaire européen

graphique des chi2 de liaison des dates aux classes (du 26 septembre 2012 à gauche au 19 octobre 2012 à droite) ${ }^{24}$.
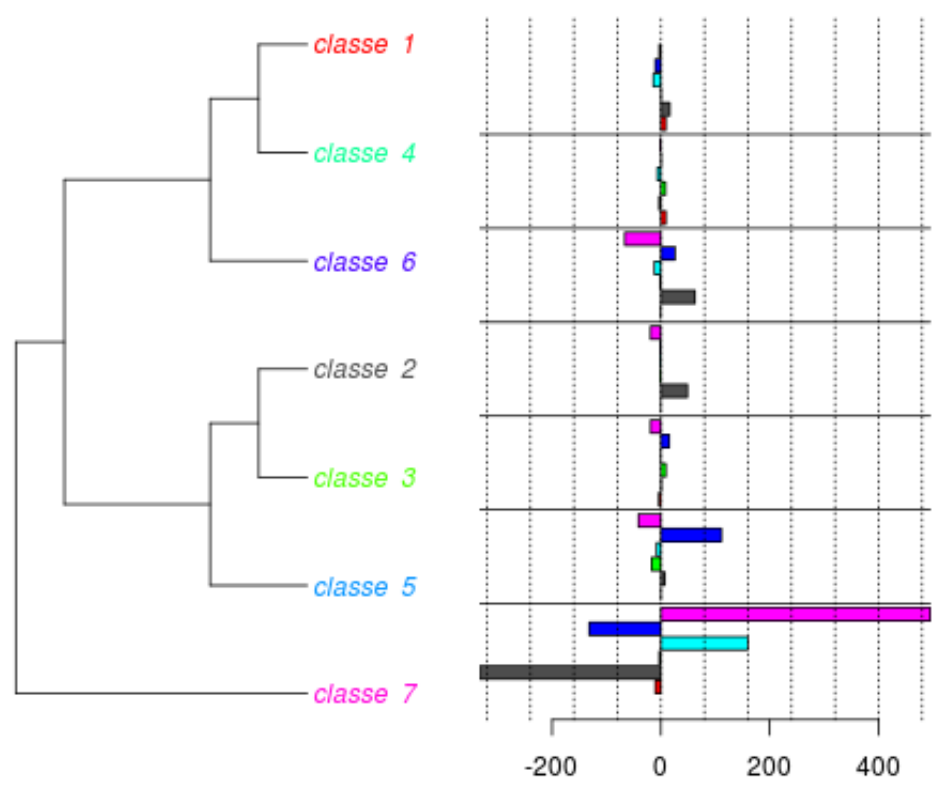

*com_msoudais

*com_quatremer

$\square$ *com_linsurge

$\square$ *com_stopausterite

- *com_cleamounette

*com_melenchon

Figure 7. Dendrogramme de la classification et projection des chi2 de liaison des communautés aux classes. Un chi2 positif indique une surreprésentation des tweets de la communauté dans la classe.

La figure 7 projette sur le dendrogramme de la classification le lien entre les communautés et les classes de discours (exprimé par un chi2). Plus le chi2 est fort, plus les tweets issus de la communauté sont surreprésentés dans la classe. Au contraire, un chi2 négatif signale une sous-représentation de la communauté dans la classe (c'est-à-dire une proportion faible de tweets issus de cette communauté à l'intérieur de la classe).

\footnotetext{
${ }^{24}$ Rappelons que le chi2 permet de mesurer un lien de dépendance statistique entre les modalités de deux variables nominales. Ici, il est utilisé pour estimer le lien entre les dates et les classes de discours ou entre les mots et les classes de discours.
} 


\section{Nikos SMYRNAIOS et Pierre RATINAUD}

La classe 1 est constituée de messages qui commentent, souvent en direct, les propos des différents hommes politiques, notamment ceux du premier ministre, lors des émissions de télévision. C'est la classe la plus volumineuse.

La classe 2 se compose essentiellement de tweets informatifs qui font le décompte de voix pour chaque camp. Son point culminant est le 9 octobre (figure 6), date du vote à l'Assemblée. Nous observons une légère surreprésentation du groupe de Jean Quatremer, composé de personnes plutôt neutres ou favorables au traité (figure 7).

La classe 3 est constituée de commentaires politiques concernant le vote à l'Assemblée, notamment des critiques virulentes et souvent sarcastiques qui sont adressées aux députés socialistes ayant approuvé le Traité. En plus des informations factuelles sur le vote des députés, cette classe contient des tweets qui critiquent la position commune entre PS et UMP. Sont également pointées les incohérences des députes socialistes qui ont voté contre le TSCG alors qu'ils avaient approuvé la loi organique sur ladite « règle d'or » budgétaire. Cette classe est la deuxième plus importante en termes de volume de tweets. Son point culminant est le 9 octobre, date du vote (figure 6). Elle est répartie de manière assez uniforme dans les différents groupes d'usagers avec une légère prééminence des communautés liées à J.-L. Mélenchon et à Clea Mounette, mais qui est statistiquement peu significative (figure 7).

La classe 4 est une critique sévère de la finance et de ses acteurs (banque, FMI) dont le comportement est lié par une relation de cause à effet aux problèmes socioéconomiques de la France (chômage, reforme de retraites, etc.).

La classe 5 est constituée essentiellement du live-tweeting des débats à l'Assemblée, avec notamment mention des différentes prises de parole des députés et particulièrement de ceux et celles qui s'opposent au Traité (Nicolas Dupont-Aignan surtout mais aussi Marc Dolez et Marion Maréchal-Le Pen). Son point culminant est le 8 octobre, veille du vote à l'Assemblée (figure 6). Nous trouvons une légère surreprésentation de cette classe auprès du groupe associé aux opposants de droite au Traité (figure 7).

La classe 6 se compose de tweets qui partagent des analyses d'experts ou d'hommes politiques sur le Traité. Elle est surreprésentée le 2 octobre, date du début des débats à l'Assemblée (figure 6), et auprès du groupe de ceux qui sont les moins critiques envers le Traité (figure 7).

Enfin, la classe 7 , quant à elle, culmine le 30 septembre, date de la manifestation à Paris (figure 6). Elle se compose d'appels à manifester et de 
Comment articuler analyse des réseaux et des discours sur Twitter :

L'exemple du débat autour du pacte budgétaire européen

témoignages depuis l'endroit de l'évènement. Elle est significativement surreprésentée auprès du groupe des opposants de gauche au Traité (figure 7).

Globalement, la répartition chronologique des classes de discours repérées avec Iramuteq est cohérente avec le déroulement des évènements qui ont ponctué le débat politique autour du Traité pendant la période étudiée: manifestation, débats parlementaires et vote. La mise en évidence de ces classes de discours peut constituer le point de départ d'une analyse de contenu qualitative, qui n'est pas l'objet de cet article mais qui serait nécessaire à une compréhension approfondie du débat. Par ailleurs, la répartition des classes parmi les différentes communautés d'usagers mises en évidence par l'analyse de graphes est également dans l'ensemble en accord avec les positionnements politiques respectifs des communautés en question. Elle permet aussi de repérer les acteurs qui occupent une position centrale dans le débat et/ou qui sont représentatifs d'un certain type de discours. Elle montre par exemple que les appels à la manifestation sont majoritairement portés par les opposants liés au Front de Gauche quand le live-tweeting des interventions des politiciens souverainistes et frontistes est significativement plus porté par la communauté des opposants de droite et d'extrême droite. Les classes de discours purement informatives (classes 2 et 6 ) sont quant à elles caractérisées par une surreprésentation de la communauté la plus proche des médias traditionnels.

\section{Conclusion}

Le sujet que nous avons analysé s'avère constituer sur Twitter un véritable débat citoyen en ligne autour d'une question politique. Les différentes analyses semi-automatiques de données que nous avons réalisées montrent plusieurs caractéristiques de ce débat qui confirment des tendances observées par d'autres recherches similaires: les échanges mêlent des messages essentiellement informatifs (comme le live-tweeting de débats télévisés et parlementaires, le décompte de voix et les analyses d'experts), mais aussi toute une gamme d'expressions sans véritable contenu informatif (ironie, critique, humour, indignation, etc.). Les interactions avec des individus précis (retweets, mentions) sont surreprésentées, ce qui montre qu'il y a bien une forme de débat structuré, notamment autour d'un petit nombre d'usagers influents, et non pas simplement une juxtaposition d'expressions individuelles. Dans le même temps, les échanges sont dominés par les opposants à travers des discours d'indignation et de dénonciation.

Enfin, d'un point de vue méthodologique, nos résultats montrent qu'il y a bien une cohérence entre d'une part les communautés d'usagers repérés par 


\section{Nikos SMYRNAIOS et Pierre RATINAUD}

l'analyse de graphes et, d'autre part, les classes de discours repérées avec Iramuteq, que porte chaque communauté. Nous pouvons conclure ainsi que cette enquête exploratoire confirme en grande partie la validité de la méthodologie que nous proposons. Dans le futur, il serait intéressant de l'appliquer sur d'autres sujets de débat sur Twitter de plus grande envergure en la complétant avec des méthodes d'enquête plus classiques en sciences humaines et sociales.

\section{Références bibliographiques}

BAYM N.K. et B. BOYD, 2012, "Socially Mediated Publicness: An Introduction ", Journal of Broadcasting \& Electronic Media, vol. 56, $\mathrm{n}^{\circ} 3$, pp. 320-329.

BOYD D.M. et N. B. ELLISON, 2007, "Social Network Sites: Definition, History, and Scholarship », Journal of Computer-Mediated Communication, vol. 13, $\mathrm{n}^{\circ} 1$, pp. 210-230.

BIDART C., A. DEGENNE et M. GROSSETTI, 2011, La vie en réseau. Dynamique des relations sociales, Paris, Presses Universitaires de France.

BLONDEL V.D., J-L. GUILLAUME, R. LAMBIOTTE et E. LEFEBVRE, 2008, «Fast unfolding of communities in large networks », Journal of Statistical Mechanics: Theory and Experiment $\mathrm{n}^{\circ} 10, \mathrm{p} .1000$.

BRUNS A. et J. BURGESS, 2011, «\#Ausvotes: how Twitter covered the 2010 Australian federal election ", Communication, Politics and Culture, vol. 44, n², pp. 37-56.

BURGESS J. et A. BRUNS, 2012, "Twitter Archives and the Challenges of 'Big Social Data' for Media and Communication Research ", M/C Journal 15, $\mathrm{n}^{\circ} 5$, $<$ http://journal.mediaculture.org.au/index.php/mcjournal/article/viewArticle/561>, dernière consultation le 11 février 2014.

CARDON D., 2010, La démocratie internet, Paris, Seuil.

CHA M., H. HADDADI, F. BENEVENUTO et K. P. GUMMADI, 2010, "Measuring User Influence in Twitter: The Million Follower Fallacy", Proceedings of the 4th International Conference on Weblogs and Social Media, May 23-26, George Washington University, Washington, DC, <http://snap.stanford.edu/class/cs224w-readings/cha10influence.pdf>, dernière consultation le 11 février 2014.

ESQUENAZI J.P, 2003, Sociologie des publics, Paris, La Découverte. 
Comment articuler analyse des réseaux et des discours sur Twitter :

L'exemple du débat autour du pacte budgétaire européen

FOUETILLOU G., 2008, « Le web et le traité constitutionnel européen. Écologie d'une localité thématique compétitive », Réseaux, n¹47, pp. 229-257.

GERLITZ C. et B. RIEDER, 2013, «Mining One Percent of Twitter: Collections, Baselines, Sampling », M/C Journal, vol. 16, $\mathrm{n}^{\circ} 2$, <http://journal.mediaculture.org.au/index.php/mcjournal/article/viewArticle/620>, dernière consultation le 11 février 2014.

LOTAN G., E. GRAEFF, M. ANANNY, D. GAFFENY, I. PEARCE et D. BOYD, 2011, " The Revolutions Were Tweeted: Information Flows during the 2011 Tunisian and Egyptian Revolutions", International Journal of Communication, vol. 5, pp. 1375-1405.

LOVINK G., 2012, Networks Without a Cause: A Critique of Social Media, Cambridge and Malden, Polity.

MAIREDER A. et J. AUSSERHOFER, 2013, "Political Discourses on Twitter: Networking Topics, Objects and People ", dans K. WELLER, A. BRUNS, J. BURGESS, M. MAHRT et C. PUSCHMANN (dir.), Twitter and Society, New York, Peter Lang.

MOSCOVICI S., 1961, La psychanalyse, son image et son public, Paris, Presses Universitaires de France.

RATINAUD P., 2009, IRaMuTeQ: interface de $R$ pour les analyses multidimensionnelles de textes et de questionnaires, $<$ http://www.iramuteq.org $>$, dernière consultation le 12 mars 2014.

RATINAUD P. et P. MARCHAND, 2012, «Application de la méthode ALCESTE à de 'gros' corpus et stabilité des «mondes lexicaux»: analyse du 'CableGate' avec IRaMuTeQ» dans Actes des 11eme Journées internationales d'Analyse statistique des Données Textuelles, Liège, Belgique, pp.835-844.

REBILLARD F., 2011, "L'étude des médias est-elle soluble dans l'informatique et la physique ? À propos du recours aux digital methods dans l'analyse de l'information en ligne ", Questions de communication, n²0, pp. 353-376.

RIEDER B., 2012, "The refraction chamber: Twitter as sphere and network », First Monday, vol. 17, $\mathrm{n}^{\circ}$ 10, <http://firstmonday.org/ojs/index.php/fm/article/view/4199/3359>, dernière consultation le 11 février 2014. 


\section{Nikos SMYRNAIOS et Pierre RATINAUD}

RIEDER B., 2010, “De la communauté à l'écume: quels concepts de sociabilité pour le 'web social' ? », tic\&société, vol. 4, $\mathrm{n}^{\circ} 1$ <http://ticetsociete.revues.org/822>, dernière consultation le 28 janvier 2014.

RIEDER B. et N. SMYRNAIOS, 2012, «Pluralisme et infomédiation sociale de l'actualité : le cas de Twitter ", Réseaux, n¹76, pp. 107-141.

ROGERS R., 2009, The End of the Virtual: Digital Methods, Amsterdam, Amsterdam University Press.

SEGERBERG A. et W. L. BENNETT, 2011, "Social Media and the Organization of Collective Action: Using Twitter to Explore the Ecologies of Two Climate Change Protests », The Communication Review, vol. 14, $n^{\circ} 3$, pp. 197-215.

SEMIOCAST, 2012, "Geolocation analysis of Twitter accounts and tweets ", <http://semiocast.com/en/publications/2012_07_30_Twitter_reaches_half_a_ billion_accounts_140m_in_the_US>, dernière consultation le 11 février 2014.

SMYRNAIOS N. et B. RIEDER, 2013, "Social infomediation of news on Twitter: A French case study", Necsus \#4_Autumn 2013_'Waste', <http://www.necsus-ejms.org/social-infomediation-of-news-on-Twitter-afrench-case-study/>, dernière consultation le 11 février 2014.

SMYRNAIOS N., 2013, "Entre bien commun et parangon publicitaire : une analyse socio-économique de Twitter» dans N. PELISSIER et G. GALLEZOT (dir.), Twitter : un monde en tout petit ?, Paris, L'Harmattan.

VEDEL T., 2012, «Les pratiques d'information politique en ligne », Communication au colloque Pluralisme de l'information : états des lieux et nouveau enjeux, Paris, 18 \& 19 septembre.

VICARI S., 2013, " Public Reasoning Around Social Contention: A Case Study of Twitter Use in the Italian Mobilization for Global Change ", Current Sociology, vol. 61, n4, pp. 474-490.

WATTS, D. J., 2004, "The 'New' Science of Networks ", Annual Review of Sociology, vol. 30, n¹, pp. 243-270. 